\title{
Herpes Gestationis Autoantibodies Recognize a 180-kD Human Epidermal Antigen
}

\author{
L. H. Morrison, R. S. Labib, J. J. Zone, L. A. Diaz, and G. J. Anhalt \\ Department of Dermatology, The Johns Hopkins Medical Institutions, Baltimore, Maryland 21205; and Division of Dermatology, \\ University of Utah, Salt Lake City, Utah 84112
}

\begin{abstract}
Herpes gestationis (HG) is a putative autoimmune bullous dermatosis of pregnancy which shares many findings with bullous pemphigoid (BP), a disease of the elderly. This study identifies for the first time the antigen detected by HG autoantibodies and compares it with that recognized by BP autoantibodies.

Sera from 16 HG and 17 BP patients, and from normal pregnant women were evaluated by immunofluorescent (IF) studies and immunoblotted against human epidermal extracts. 89\% of HG sera with circulating antibodies by IF recognized a 180-kD protein by immunoblotting. $71 \%$ of $B P$ sera recognized a $240-\mathrm{kD}$ band, but $47 \%$ detected a $180-\mathrm{kD}$ protein that comigrated with the antigen detected by HG sera. None of the control sera recognized any specific bands.

These findings suggest that the $180-\mathbf{k D}$ epidermal protein may be the antigen detected by the HG factor and they also define immunologic similarities between HG and BP.
\end{abstract}

\section{Introduction}

Herpes gestationis $(\mathrm{HG})^{1}$ is a rare, subepidermal bullous dermatosis of pregnancy and the postpartum period that characteristically begins in the second trimester and usually resolves within 3 mo after delivery $(1,2)$. Immunopathologic features of the disease include linear deposition of C3 and, occasionally, IgG along the basement membrane zone (BMZ) of perilesional skin by direct immunofluorescence (IF), and, frequently, presence of a circulating IgG autoantibody directed against a BMZ antigen that avidly fixes complement $(3,4)$. This was originally referred to as the " $\mathrm{HG}$ factor" before it was recognized as an IgG autoantibody (5-7). Although this autoantibody is commonly detectable in HG patient sera, little is known of the antigen to which it binds.

Address all correspondence to Dr. Lynne H. Morrison, Department of Dermatology, The Johns Hopkins Hospital, 600 North Wolfe Street, Baltimore, MD 21205.

Received for publication 12 January 1988 and in revised form 25 February 1988.

1. Abbreviations used in this paper: BMZ, basement membrane zone; BP, bullous pemphigoid; HG, herpes gestationis; IF, immunofluorescence.

J. Clin. Invest.

(c) The American Society for Clinical Investigation, Inc. $0021-9738 / 88 / 06 / 2023 / 04 \$ 2.00$

Volume 81, June 1988, 2023-2026
HG shares several features with bullous pemphigoid (BP), a blistering disease of the elderly. Both diseases clinically demonstrate urticarial plaques and tense bullae, and histologically show subepidermal blisters $(3,8)$. They both also commonly have autoantibodies of the IgG class directed against an antigen in the epidermal BMZ. IF studies reflect this, showing linear deposition of IgG and $\mathrm{C} 3$ along the $\mathrm{BMZ}$ in $\mathrm{BP}$ and heavy linear $\mathrm{BMZ}$ deposition of $\mathrm{C} 3$ with weak or undetectable IgG in $H G(3,8)$. These similarities have lead to the concept that these two diseases may be related or that they are the same disease occurring in different populations.

The molecular weight of the BP antigen(s) has been determined by both immunoprecipitation and immunoblotting. Current evidence suggests that there may be more than one antigen recognized by BP sera. Stanley et al. have immunoprecipitated a $220-\mathrm{kD}$ protein from epidermal cell culture, and subsequently identified a $220-240-k D$ protein derived from normal human epidermis using immunoblot analysis $(9,10)$. By immunoblotting 28 BP sera against normal human epidermis, Labib et al. (11) have found that BP antibodies most frequently recognize a $240-\mathrm{kD}$ or a $180-\mathrm{kD}$ protein. Although the molecular weight of the BP antigen(s) has been determined by the above techniques, the HG antigen has not yet been identified. The purpose of this study therefore was to characterize the antigen detected by $\mathrm{HG}$ autoantibodies and to compare it with the antigen recognized by BP antibodies.

\section{Methods}

Source of sera. We obtained 16 sera from 11 patients with HG. The diagnosis at the time of presentation was documented clinically, by histology and by direct and/or indirect complement fixation IF. These procedures have been described elsewhere in detail $(12,13)$. Some of the sera were collected sequentially from individual patients both during active disease and later, after resolution of the eruption.

Control sera were obtained from 26 healthy pregnant women in their third trimester. Sera were also obtained from 17 BP patients through the clinical immunopathology laboratory at The Johns Hopkins Medical Institutions, Department of Dermatology. These patients fulfilled the clinical, histologic, direct and indirect IF criteria for that disease (8).

IF studies. Each of the HG, BP, and control sera was evaluated for presence of HG factor activity by indirect complement fixation IF studies (HG factor assay). The sera were first heat inactivated at $56^{\circ} \mathrm{C}$ for 30 min to remove complement activity. Normal human skin was incubated with the sera diluted 1:10 in PBS for $30 \mathrm{~min}$ at room temperature. After the skin was washed, it was incubated with normal human sera as a complement source, which was diluted 1:10 in M-199 (Flow Laboratories, Inc., McLean, VA), and then, after being washed again, it was incubated with FITC-conjugated anti-human C3. The speci- 
mens were examined with a fluorescent microscope (Olympus Corp. of America, New Hyde Park, NY). The HG factor assays were done at least twice on each sera.

Routine indirect IF studies were performed on all BP sera as previously described (12). Either monkey esophagus or normal human skin was used as a substrate, and FITC-conjugated monospecific antibodies to IgG, IgM, IgA, and C3 were employed.

Fluorescein/protein ratios for the conjugates used in IF studies were as follows: IgG, 3.74; IgM, 3.75; IgA, 4.72; C3, 3.76 mg/g (Cappel Laboratories, Malvern, PA).

Western immunoblotting. Skin was obtained from surgical or autopsy specimens, incubated overnight in 2 mM EDTA in PBS, with 2 $\mathrm{mM}$ PMSF at room temperature. The epidermis was then separated from the dermis. An indirect complement fixation IF, done on skin separated in this way, showed deposition of immunoreactants only on the roof of the split.

The epidermis was cut into small fragments and homogenized on ice in a solution containing $1 \%$ SDS in $0.01 \mathrm{M}$ Tris/ $\mathrm{HCl}$ buffer, $\mathrm{pH} 7.6$, $5 \%$ beta-mercaptoethanol, and a wide range of protease inhibitors including $2 \mathrm{mM}$ PMSF, $10 \mathrm{mM}$ EDTA, $10 \mu \mathrm{g} / \mathrm{ml}$ each of leupeptin, antipain, chymostatin, and pepstatin. Homogenization was carried out with a Polytron (Brinkmann Instruments Co., Westbury, NY). The suspension was then boiled for $3 \mathrm{~min}$, centrifuged at $3,500 \mathrm{rpm}$ for 10 min, and the resulting supernatant was either used immediately or frozen at $-70^{\circ} \mathrm{C}$ until ready for use.

The epidermal proteins were separated using 7\% SDS-PAGE and a minicell (Bio-Rad Laboratories, Richmond, CA). One pair of adjacent lanes containing molecular weight standards and epidermal extract was stained with Coomassie Brilliant Blue R-250. The unstained portion of the gel was electrophoretically transferred to a nitrocellulose matrix (Millipore Corp., Bedford, MA) using a Transblot apparatus (Bio-Rad Laboratories). The transferred proteins were detected using fast green FCF. The nitrocellulose was cut into strips, each containing a lane of molecular weight standards and a lane of epidermal extract. They were first incubated in $5 \%$ low fat milk in a washing buffer consisting of $0.15 \mathrm{M} \mathrm{NaCl}, 0.05 \%$ Tween-20, in $0.01 \mathrm{M}$ Tris buffer. After three rinsings in washing buffer, they were incubated in either HG, BP, or control sera, diluted 1:100 in washing buffer. After triplicate rinsing they were incubated with biotinylated anti-human IgG (Bio-Rad Laboratories) diluted 1:1,000 then, again rinsed in triplicate and incubated with streptavidin (Bio-Rad Laboratories) diluted 1:1,000. All incubations were done for $1 \mathrm{~h}$ at $37^{\circ} \mathrm{C}$. Finally, the strips were washed first with three changes of washing buffer, then with $\mathbf{5 0}$ $\mathrm{mM}$ Tris- $\mathrm{HCl}, \mathrm{pH} \mathrm{7.6,} \mathrm{at} \mathrm{rocm} \mathrm{temperature.} \mathrm{They} \mathrm{were} \mathrm{then} \mathrm{incu-}$

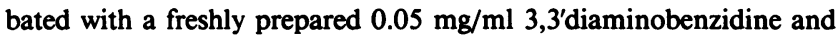

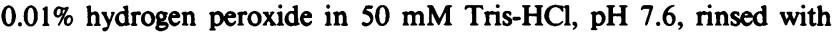
water, and dried.

\section{Results}

$I F$. We found that 9 of the 16 sera obtained from the HG patients had complement fixing anti-BMZ autoantibody demonstrable by indirect complement fixation IF (i.e., HG factor activity). Eight of these nine sera were taken from patients who had active disease at the time the sera was obtained. The clinical status of the remaining patient was unavailable.

All 17 sera from BP patients had anti-BMZ antibodies of the IgG class detectable with routine indirect IF in titers ranging from 1:80-1:2,560. 14 of the $17(76 \%)$ sera had complement fixing anti-BMZ antibodies as detected by indirect complement fixation IF. None of the 26 control sera from normal pregnancies showed HG factor activity.

Immunoblotting. Of the nine HG sera that were HG factor positive, eight $(89 \%)$ had autoantibodies that recognized a 180-kD human epidermal protein (Table I). One sera detected a $240-\mathrm{kD}$ band in addition to the $180-\mathrm{kD}$ protein and one did
Table I. Detection of HG Factor by IF and 180/240-kD Antigens by Immunoblotting

\begin{tabular}{lccc}
\hline & HG factor & 180 kD & 240 kD \\
\hline Serum 1 & + & + & + \\
Serum 2 & + & + & - \\
Serum 3 & + & + & - \\
Serum 4 & + & + & - \\
Serum 5 & + & + & - \\
Serum 6 & + & + & - \\
Serum 7 & + & + & - \\
Serum 8 & + & + & - \\
Serum 9 & + & - & - \\
Serum 10 & - & + & - \\
Serum 11 & - & - & - \\
Serum 12 & - & - & - \\
Serum 13 & - & - & - \\
Serum 14 & - & - & - \\
Serum 15 & - & - & - \\
Serum 16 & - & - & - \\
\hline
\end{tabular}

not show any specific bands. Immunoblot analysis using the seven remaining sera, which did not show HG factor activity by IF techniques, revealed that one did recognize the $180-\mathrm{kD}$ band, but the remaining six $(86 \%)$ detected no specific bands. None of the 26 control sera from pregnant women recognized either the 180 - or $240-\mathrm{kD}$ epidermal protein bands.

Fig. 1 shows representative results from immunoblotting. The $180-\mathrm{kD}$ band is clearly seen in the three immunoblots that have been incubated with HG sera, but is absent in the control immunoblot. A second, fainter band immediately beneath the $180-\mathrm{kD}$ protein, with an apparent molecular weight of $160 \mathrm{kD}$, is seen in one of the immunoblots. This band was detected with epidermal extracts which had been stored for longer than a few weeks before use, but not in newly prepared extracts made from "fresh" surgical specimens. Its appearance was accompanied by a loss in intensity of the $180-\mathrm{kD}$ band and its presence was not restricted to any particular extract or serum. We believe that this lower molecular weight band is likely due to proteolysis of the $180-\mathrm{kD}$ protein and does not represent a separate antigen.

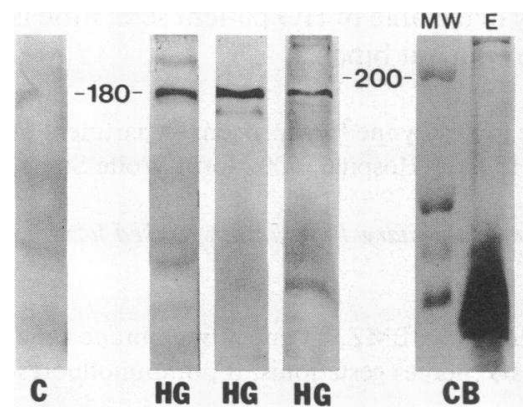

Figure 1. Epidermal antigens recognized by sera from patients with HG. On the right is a Coomassie Blue-stained SDS-PAGE gel $(C B)$ showing one lane of molecular weight standards $(M W)$, with the 200-kD band marked, and a lane of total epidermal extract $(E)$. The center three lanes (labeled HG) are immunoblots of this extract that have been incubated with HG sera. The lane on the left $(C)$ is an immunoblot that has been incubated with sera from a normal pregnant woman. The three HG sera strongly recognize the $180-\mathrm{kD}$ epidermal antigen. The control does not recognize any specific bands. 
The second aim of the study was to compare the antigens recognized by antibodies from HG and BP sera. We have performed immunoblot analysis on 17 BP sera with the following results: $12(71 \%)$ detected a $240-\mathrm{kD}$ protein, $8(47 \%)$ sera recognized the $180-\mathrm{kD}$ band (4 of which also recognized the 240 $\mathrm{kD}$ band), and $1(6 \%)$ did not detect any specific protein.

Fig. 2 shows immunoblots incubated with BP sera and others incubated with HG sera for comparison. Both HG sera strongly recognize a $180-\mathrm{kD}$ protein band. The BP serum also strongly detects the $180-\mathrm{kD}$ protein, which comigrated with the $180-\mathrm{kD}$ protein detected by the HG sera. It did not detect a 240-kD epidermal protein, which was more clearly detectable in many other BP sera. The $160-\mathrm{kD}$ band, which most likely represents a breakdown product of the $180-\mathrm{kD}$ band, is more prominent in these immunoblots.

\section{Discussion}

Many pieces of evidence suggest that HG may be an organ-specific autoimmune disease. These include the presence of tissue-bound and circulating autoantibodies, and the development of similar lesions in neonates, which are attributed to transplacental transfer of autoantibody from the affected mother (4). There has been speculation about the relationship of this disorder of pregnancy to BP, a similar autoimmune skin disease of the elderly. Better understanding of this association has been hampered partly by the failure to define the antigens to which the low titer autoantibodies (the "HG factor") are directed.

Using sera from patients with HG, we have shown that $89 \%$ of sera that possess HG factor activity (as shown by IF techniques) also recognize a $180-\mathrm{kD}$ epidermal protein by immunoblotting. Of those sera that have no HG factor activity by IF criteria, $86 \%$ showed no specific bands by immunoblotting. This shows a remarkably high correlation between the presence of the HG factor, a complement fixing IgG characteristic of HG, and the detection of a $180-\mathrm{kD}$ epidermal protein by immunoblotting $(P<0.005$, by chi-square analysis). This correlation strongly suggests that a $180-\mathrm{kD}$ epidermal protein is the antigen recognized by the HG factor. This $180-\mathrm{kD}$ protein was not detected by any of the sera from normal pregnant

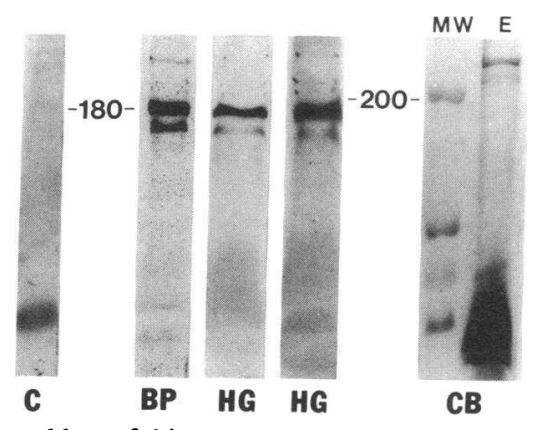

Figure 2. Comparison of epidermal antigens recognized by sera from patients with $\mathrm{HG}$ and BP. On the right is a Coomassie Blue-stained SDS-PAGE gel $(C B)$, showing one lane of molecular weight standards $(M W)$ and one lane of total epidermal extract $(E)$. Two immunoblots of this extract that had been incubated with HG sera (labeled $H G$ ), and an immunoblot that had been incubated with BP sera (labeled $B P$ ) are shown to the left. The BP sera recognize the $180-\mathrm{kD}$ epidermal antigen which comigrated with the $180-\mathrm{kD}$ protein detected by the HG sera. A proteolytic breakdown product of the 180 $\mathrm{kD}$ band with an apparent molecular weight of $160 \mathrm{kD}$ is seen in these blots. The immunoblot on the left has been incubated with control sera $(C)$ and does not show any specific bands. women, which establishes that the production of autoantibodies to this protein is specific to HG and is not a feature of a normal pregnancy. It is not, however, entirely unique to this disease and is detected by a minority of patients with BP.

One of our HG sera, which did not have HG factor activity, did detect the $180-\mathrm{kD}$ band by immunoblotting. We think that this can be attributed to the greater sensitivity of immunoblotting, when employed with the biotin avidin system, as compared with the indirect complement fixation IF technique. We also had a single HG sera that did have HG factor activity but failed to recognize the $180-\mathrm{kD}$ protein by immunoblotting. This is most likely related to the denaturation of the HG antigen that inevitably occurs during extraction and immunoblotting. Exposure to $1 \%$ SDS, an ionic detergent that disrupts the tertiary configuration of proteins, and reduction by beta mercaptoethanol will commonly produce enough denaturation of antigen with the result that predicted antigen-antibody reactions may not occur. In view of this phenomenon it is gratifying that $89 \%$ of those patients' autoantibodies that bind to native antigen in IF procedures apparently retain their ability to detect the denatured antigen in Western blots.

We found that although most BP sera recognized a 240-kD protein by immunoblotting, $47 \%$ recognized a $180-\mathrm{kD}$ protein either as a single finding or in addition to the $240-\mathrm{kD}$ band. Detection of these bands by BP sera is in agreement with what has been reported in the literature (9-11). Detection of a 180 $\mathrm{kD}$ epidermal protein by both HG and BP sera establishes a degree of similarity between these two putative autoimmune diseases that had been the subject of previous speculation. It appears that a subset of BP antibodies may recognize either the same antigen or a different epitope on the same molecule as do HG antibodies. This finding provides further support for the concept that these diseases are related.

Studies are in progress to further characterize this antigen. It is established that BP autoantibodies bind specifically to the hemidesmosome of squamous epithelia (14-16) and our laboratory and others have developed MAbs to a $180-\mathrm{kD}$ antigen that also specifically bind only to the hemidesmosomes of squamous epithelia $(17,18)$. It may be that HG patients' autoantibodies recognize this same antigen, but this has yet to be established.

In summary, this study provides the first biochemical characterization of the antigen recognized by autoantibodies in the putative autoimmune disease, herpes gestationis. Additionally, it provides basic information demonstrating that $\mathrm{HG}$ and $\mathrm{BP}$ are immunologically related disorders.

\section{Acknowledgments}

We would like to thank Drs. R. Gammon and J. Shornick for providing sera for this study.

This work was supported in part by U. S. Public Health Service grants R01-AR32599, R01-AR32490, K04-AR01686, R01-AR32079, R0-AM35378-01, and T32-AR07324 from the National Institutes of Health, and by the Veteran's Administration Medical Center, Salt Lake City (J. J. Zone).

\section{References}

1. Shornick, J. K., J. L. Bangert, R. G. Freeman, and J. N. Gilliam. 1983. Herpes gestationis: clinical and histologic features of twentyeight cases. J. Am. Acad. Dermatol. 8:214-224. 
2. Carruthers, J. A. 1978. Herpes gestationis: clinical features of immunologically proved cases. Am. J. Obstet. Gynecol. 131:865-867.

3. Eaglestein, N. F., G. J. Anhalt, H. P. Patel, and L. A. Diaz. 1987. Herpes gestationis. In Immunopathology of the Skin. E. Beutner, T. Chorzelski, and V. Kumar, editors. John Wiley and Sons, New York. 371-378.

4. Jordon, R. E., K. G. Heine, G. Tappeiner, L. L. Bushkell, and T. T. Provost. 1976. The immunopathology of Herpes gestationis. Immunofluorescence studies and characterization of the "HG factor." J. Clin. Invest. 57:1426-1433.

5. Provost, T. T., and T. B. Tomasi, 1973. Evidence for complement activation via the alternate pathway in skin disease I. Herpes gestationis, systemic lupus erythematosus, and bullous pemphigoid. $J$. Clin. Invest. 52:1779-1787.

6. Katz, S. I., K. C. Hertz, and H. Yaoita. 1976. Herpes gestationis. Immunopathology and characterization of the HG factor. J. Clin. Invest. 57: 1434-1441.

7. Carruthers, J. A., and A. R. Ewins. 1978. Herpes gestationis: studies on the binding characteristics, activity and pathogenetic significance of the complement-fixing factor. Clin. Exp. Immunol. 31:38-44.

8. Chorzelski, T. P., S. Jablonska, and E. Beutner. 1987. Bullous pemphigoid: relationship of immunopathology to clinical findings. In Immunopathology of the Skin. E. Beutner, T. Chorzelski, and V. Kumar, editors, John Wiley and Sons, New York. 337-352.

9. Stanley, J. R., P. Hawley-Nelson, S. Yuspa, E. Shevach, and S. Katz. 1981. Characterization of bullous pemphigoid antigen: a unique basement membrane protein of stratified squamous epithelia. Cell. 24:897-903.

10. Stanley, J. R., D. T. Woodley, and S. I. Katz. 1984. Identification and partial characterization of pemphigoid antigen extracted from normal human skin. J. Invest. Dermatol. 82:108-111.
11. Labib, R. S., G. J. Anhalt, H. P. Patel, D. F. Mutasim, and L. A. Diaz. 1986. Molecular heterogeneity of the bullous pemphigoid antigens as detected by immunoblotting. J. Immunol. 136-1231-1235.

12. Jordon, R. E., E. H. Beutner, E. Witebsky, G. Blumenthal, W. L. Hale, and W. F. Lever, 1967. Basement zone antibodies in bullous pemphigoid. J. Am. Med. Assoc. 200:751-756.

13. Jordon, R. E., W. M. Sams, Jr., and E. H. Beutner. 1969. Complement immunofluorescent staining in bullous pemphigoid. $J$. Lab. Clin. Med. 74: 548-556.

14. Mutasim, D. F., Y. Takahashi, R. S. Labib, G. J. Anhalt, H. P. Patel, and L. A. Diaz. 1985. A pool of bullous pemphigoid antigen(s) is intracellular and associated with the basal cell cytoskeleton-hemidesmosome complex. J. Invest. Dermatol. 84:47-53.

15. Regnier, M., P. Vaigot, S. Michel, and M. Prunieras. 1985. Localization of bullous pemphigoid antigen (BPA) in isolated human keratinocytes. J. Invest. Dermatol. 85:187-190.

16. Westgate, G. E., A. C. Weaver, and J. R. Couchman. 1985. Bullous pemphigoid antigen localization suggests an intracellular association with hemidesmosomes. J. Invest. Dermatol. 84:218-224.

17. Jones, J. C. R. 1987. Characterization of components of the hemidesmosome. J. Cell Biol. 105: 263a. (Abstr.)

18. Burek, C. L., E. Avalos, N. R. Rose, D. F. Mutasim, R. S. Labib, H. P. Patel, G. J. Anhalt, and L. A. Diaz. 1985. Rat-mouse hybridomas producing monoclonal antibodies reactive with a basal cell hemidesmosome antigen similar to human bullous pemphigoid autoantibodies. Clin. Res. 33:297a. (Abstr.)

19. Ortonne, J. P., B. L. Hsi, P. Verrando, F. Bernerd, G. Pautrat, A. Pisani, and C.-J. G. Yeh. 1987. Herpes gestationis factor reacts with the amniotic epithelial basement membrane. Br. J. Dermatol. 117:147-154. 